\title{
Forecasting Financial Risk using Quantum Neural Networks
}

\author{
Abdelali El Bouchti ${ }^{1,2}$, Younes Tribis ${ }^{2,3}$, Tarik Nahhal ${ }^{1}$, Chafik Okar ${ }^{4}$ \\ ${ }^{1} \mathrm{FS}$, Hassan 2nd University, Casablanca, Morocco \\ ${ }^{2}$ Institute for Forecasting and Futuristics \\ ${ }^{3}$ FST, Hassan 1st University, Settat, Morocco \\ ${ }^{4}$ ENSA, Hassan 1st University, Berrechid, Morocco \\ a.elbouchti@gmail.com,y.tribis@uhp.ac.ma,t.nahhal@fsac.ac.ma,okar.chafik@gmail.com
}

ABSTRACT: There has been enormous attention in quantum algorithms for reinforcing machine learning (ML) algorithms. In the current paper, we present quantum neural networks (QNNS) and a method of training which is well in quantum system and is improved with momentum accession and parameter self adaptive algorithm, and we build a new financial risk forecasting model. We apply this model to the empirical research on the financial risk forecasting of some Moroccan companies. Then we will compare the findings with the standard artificial neural network (ANNs).

Keywords: Financial Risk, Neural Networks, Quantum Computing, Artificial Neural Networks

Received: 24 May 2019, Revised 29 June 2019, Accepted 25 July 2019

DOI: $10.6025 /$ jisr/2019/10/3/97-104

C2019 DLINE. All rights reserved

\section{Introduction}

Nowadays, forecasting financial risk has become one of the main increase areas of probability and statistical modelling. When financial risk is aforesaid one heads for thinking of portfolio management, pricing of options and other financial instruments. Financial risk is also called financial crisis and financial distress, which will often make the company bankrupt. Financial risk prediction is what predicts feasibility of financial crisis with statistics and so on for companies by analyzing the company's financial report and relevant materials. The earliest researcher in financial risk prediction is Fitzpatrick, an American scholar (1932). In the subsequent research, many scholars applied multidimensional Probit model and artificial natural network to construct financial risk prediction models with better forecast ability. Odom firstly began to use the artificial neuron network to carry on the exploration of financial risk prediction in 1990.

Quantum neural networks have been proposed [1], but very few of these proposals have attempted to provide an indepth method of training them. Most either do not mention how the network will be trained or simply state that they use a standard gradient descent algorithm. This assumes that training a quantum neural network will be straightforward and analogous to 
classical methods. While some quantum neural networks seem quite similar to classical networks [2], others have proposed quantum networks that are vastly different $[3,4,5]$. Several different network structures have been proposed, including lattices [6] and dots [4]. Several of these networks also employ methods which are speculative or difficult to do in quantum systems [7, 8]. These significant differences between classical networks and quantum neural networks, as well as the problems associated with quantum computation itself, require us to look more deeply at the issue of training quantum neural networks. Furthermore, no one has done empirical testing on their training methods to show that their methods work with real-world problems.

It is an open question what advantages a quantum neural network (QNN) would have over a classical network. It has been shown that QNNs should have roughly the same computational power as classical networks [7]. Other results have shown that QNNs may work best with some classical components as well as quantum components [2]. Machine learning has been presented as one of the key applications for near-term quantum technologies, given its high commercial value and wide range of applicability. In this work, we introduce the quantum neural networks: a hybrid quantum-classical framework with the potential of tackling high-dimensional real-world machine learning datasets on continuous variables.

Quantum searches can be proven to be faster than comparable classical searches. We leverage this idea to propose a new training method for a simple QNN. This paper details such a network and how training could be done on it. Results from testing the algorithm on several real-world problems show that it works.

This paper propose a new training method for a simple QNN and it describes such a quantum neural network (QNN) and how training could be done on it. Findings from testing the algorithm on several Moroccan companies show that it works. Furthermore, we show and prove that QNN is faster than comparable classical neural networks. This article proposes QNN model. By using rough set to draw regulation from data sample book and adding rough nerve cell to membership layer of QNN, the number of regulation decreases and network training speeds up. At the same time, the training algorithm of QNN is enhanced with momentum accession and parameter self adaptive algorithm to fit training request of QNN, to effectively evade "dark box operation" in distilling regulation, and to make network training speed up. Finally, we apply this method to empirical analysis on forecasting financial risk of Moroccan companies and have realized good results.

\section{Financial Performance and Financial Risk Management}

Most firms perform the primary elements of risk management. However, companies with more mature risk practices do better financially. They outperform the other firms by making powerful decisions, more efficiently deploying rare resources and decreasing their exposure to negative events. Firms around the world have made fundamental investments, processes and technology to help control business risk. Historically, these risk investments have focused on financial controls and regulatory compliance. Effective risk management starts at the top with clarity around risk strategy and governance. It is crucial that the proper accountability exist at the executive levels.

In binding the risk management practices and financial performance for companies, the mean scores of each risk management practices will be corresponded to the ROA and ROE. Risk management practices will be explained by risk management environment, policies and procedures, risk measurement practices, risk mitigation practices, risk monitoring practices and internal control practices. The result of relation between $\mathrm{ROA} / \mathrm{ROE}$ and all risk management practices will be used to show whether there is any correlation between ROA and risk measurement practices and also to explain how strongly the variables correlate.

\subsection{Financial Performance}

Financial performance is a measure of companies' policies and operations in monetary terms. It is a common measure of a company's total operation health over a given duration, and can be used to compare similar firms across the same industry or to compare industries or sectors in collection. There are many different ways to measure a firm's financial performance. This may be reflected in the firm's ROI among others and is a subjective measure of how a firm can use assets from its primary mode of business and generate revenues. According to [5], the financial industry, like other industries is in business to earn profits by selling its products. To maximize the profits, financial institutions develop new products to satisfy their own needs as well as those of their customers. Companies are finding that many of the old ways of doing business were no longer profitable; the products and services they had been offering to the market were no longer selling [6]. To survive in the new economic environment, companies have to research and develop new products and services that would mitigate financial risks and improve profitable [7].

98 Journal of Information Security Research Volume 10 Number 3 September 2019 


\subsection{Financial Risk Management Methods}

Financial risks are only one category of a broad field of risks. Furthermore financial risks can be classified into three subclasses credit risk, liquidity risk, and market risk. Market risk can be classified into four broad classes, foreign currency, interest rate, commodity, and equity risk. For financial risk management there are many different kinds of definitions. Some researchers define it whether very broadly or narrowly which leads that there is no globally accepted definition of financial risk management [8]. However financial risk is such a complex and extensive concept that financial risk-management practitioners need often specialize themselves only to certain part of financial risk management as for instance foreign exchange risk. The authors in [9] have relatively narrow view to risk management; he finds risk management as the risk handling process. Furthermore in [10] view risk management leads to avoiding, reducing, transferring, sharing or taking the risk. Also it is good to notice that risk management is a very broad term due to the wide range of risks and thus there are several categories of risk management as financial risk management operational risk management, supply chain risk management [5].

\section{Quantum Computation Background}

Particular required ideas that shape the basis for the study of quantum computation are reviewed in [9].

\subsection{Linear Superposition}

Linear superposition is closely related to the familiar mathematical principle of linear combination of vectors. Quantum systems are described by a wave function $\psi$ that exists in a Hilbert space. The Hilbert space has a set of states, $\left|\phi_{i}\right\rangle$, that form a basis, and the system is described by a quantum state $\left|\psi>=\Sigma_{i} c_{i}\right| \phi_{i}>$. $\mid \psi>$ is said to be coherent or to be in a linear superposition of the basis states $\mid \phi_{i}>$, and in general the coefficients $c_{i}$ are complex. A postulate of quantum mechanics is that if a coherent system interacts in any way with its environment, the superposition is destroyed. This loss of coherence is governed by the wave function $\psi$. The coefficients $c_{i}$ are called probability amplitudes, and $\left|c_{i}\right|^{2}$ gives the probability of $|\psi\rangle$ being measured in the state $\mid \phi_{i}>$. Note that the wave function $\psi$ describes a real physical system that must collapse to exactly one basis state. Therefore, the probabilities governed by the amplitudes $c_{i}$ must sum to unity. A two-state quantum system is used as the basic unit of quantum computation. Such a system is referred to as a quantum bit or qubit and naming the two states $\mid 0>$ and $\mid 1>$, it is easy to see why this is so.

\subsection{Operators}

Operators on a Hilbert space describe how one wave function is changed into another and they may be represented as matrices acting on vectors (the notation $\mid \cdot i$ indicates a column vector and the $h \cdot \mid$ a [complex conjugate] row vector). Using operators, an eigenvalue equation can be written $A\left|\phi_{i}>=a_{i}\right| \phi_{i}>$, where $a_{i}$ is the eigenvalue. The solutions $\mid \phi_{i}>$ to such an equation are called eigenstates and can be used to construct the basis of a Hilbert space as discussed in the last sub-section. In the quantum formalism, all properties are represented as operators whose eigenstates are the basis for the Hilbert space associated with that property and whose eigenvalues are the quantum allowed values for that property. It is important to note that operators in quantum mechanics must be linear operators and further that they must be unitary.

\subsection{Interference}

Interference is a familiar wave phenomenon. Wave peaks that are in phase interfere constructively while those that are out of phase interfere destructively. This is a phenomenon common to all kinds of wave mechanics from water waves to optics. The well known double slit experiment demonstrates empirically that at the quantum level interference also applies to the probability waves of quantum mechanics. The wave function interferes with itself through the action of an operator - the different parts of the wave function interfere constructively or destructively according to their relative phases just like any other kind of wave.

\subsection{Entanglement}

Entanglement is the potential for quantum systems to exhibit correlations that cannot be accounted for classically. From a computational standpoint, entanglement seems intuitive enough - it is simply the fact that correlations can exist between different qubits - for example if one qubit is in the $\mid 1>$ state, another will be in the $\mid 1>$ state. However, from a physical standpoint, entanglement is little understood. The questions of what exactly it is and how it works are still not resolved. What makes it so powerful (and so little understood) is the fact that since quantum states exist as superpositions, these correlations exist in superposition as well. When coherence is lost, the proper correlation is somehow communicated between the qubits, and it is this "communication" that is the crux of entanglement. Mathematically, entanglement may be described using the density matrix 
formalism. The density matrix $\rho_{\psi}$ of a quantum state $\mid \psi>$ is defined as $\rho_{\psi}=|\psi><\psi|$.

Quantum computation can be defined as representing the problem to be solved in the language of quantum states and then producing operators that drive the system (via interference and entanglement) to a final state such that when the system is observed there is a high probability of finding a solution.

\subsection{Quantum Search}

One of the best known quantum algorithms searches an unordered database quadratically faster than any classical method [12, 13]. The algorithm begins with a superposition of all $N$ data items and depends upon an oracle that can recognize the target of the search. Classically, searching such a database requires $O(N)$ oracle calls; however, on a quantum computer, the task requires only $O(\sqrt{N})$ oracle calls. Each oracle call consists of a quantum operator that inverts the phase of the search target. An "inversion about average" operator then shifts amplitude towards the target state. After $\pi / 4 * \sqrt{N}$ repetitions of this process, the system is measured and with high probability, the desired datum is the result.

\section{Developing a Quantum Neural Network Model}

We would like a QNN with features that make it easy for us to model, yet powerful enough to leverage quantum physics. We would like our QNN to use known quantum algorithms and gates, have weights which we can measure for each node, work in classical simulations of reasonable size and to be able to transfer knowledge to classical systems.

We propose a QNN that operates much like a classical ANN composed of several layers of perceptrons - an input layer, one or more hidden layers and an output layer. Each layer is fully connected to the previous layer. Each hidden layer computes a weighted sum of the outputs of the previous layer. If this is sum above a threshold, the node goes high, otherwise it stays low. The output layer does the same thing as the hidden layer(s), except that it also checks its accuracy against the target output of the network. The network as a whole computes a function by checking which output bit is high. There are no checks to make sure exactly one output is high. This allows the network to learn data sets which have one output high or binary-encoded outputs.

\subsection{Quantum Neural Network Model}

There are many approaches to the development of QNN models, proposed in [1] and [2]. These models focus on different aspects of quantum computations and neural processing. In quantum computing, As the smallest unit of information, a quantum bit or qubit is a quantum system whose states lie in a two dimensional Hilbert space. Like bits in classical computers, qubits labeled and express one bit of information: corresponds to the bit 0 of classical computers, and to the bit 1. Qubit state expresses a coherent superposition of states:

$$
|\psi>=\alpha| 0>+\beta \mid 1>,\left(|\alpha|^{2}+|\beta|^{2}=1\right)
$$

Where $\alpha$ and $\beta$ specify the probability of the corresponding states.

Quantum gate which includes the characteristics of quantum computing is the basis for physical implementation of quantum computing. The universal logic sets are included in the quantum logic. Similar with classic bit, the basic gate can form arbitrary quantum gates and complete quantum state of some of the logic of transformation. An element based on the 1 bit phase-shift gate and 2 bit controlled-Not gate in quantum dynamics are taken as a activation function in Neural Networks. To facilitate application, the following form of complex function is given to express the quantum state:

$$
f(\theta)=e^{i \theta}=\cos \theta+i \sin \theta
$$

$i=\sqrt{-1}$ is imaginary unit, $\theta$ is quantum phase, the probability amplitude of $\mid 0>$ is the real part of complex function, the probability amplitude of $\mid 1>$ is the imaginary part of complex function. Then a quantum state can be described as:

$$
|\psi>=\cos \theta| 0>+\sin \theta \mid 1>
$$

Quantum neuron model based on 1 bit phase-shift gate and 2 bit controlled-Not gate. 
$x_{i}$ is the $i^{t h}$ input to the quantum states of the neurons, $\theta_{i}$ is the phase transfer coefficient of weight, $\lambda$ is the threshold coefficient, $\delta$ is the phase control factor, $z$ is output state, $\arg (u)$ is the phase of $u$, function $f$ is defined as the formula $(1), g(x)$ is sigmoid function.

Quantum Neural Network discussed in this paper is a combination of the qubit neuron and multi-layered feed-forward neural networks.

Back Propagation (BP) algorithm is one of algorithm widely used in the neural network (NN) training. But BP algorithm has some unavoidable defects such as slow speed in training (especially for large training samples), liable to get into local minimum, and so on. Although some improved BP algorithm is given, these algorithms can hardly avoid the BP algorithm getting into local minimum. Considering of the ability of Genetic Algorithm (GA)'s global searching [3,4,5], we use improved GA to train quantum neural network architecture to avoid it getting into local minimum and obtain effective result.

\subsection{Problem Formulation}

The basic idea of QNN model based on rough set theory is to handle the input of neural network with knowledge reduction of rough set theory. Specific processes are as following:

- Step 1: The Rough set theory mainly studies data structure composed of object aggregation and property aggregation. Usually this data structure is called decision systems (table).

An information system (table) $S$ is the tuple $S=\left(U, A t,\left\{V_{a} \mid a \in A t\right\},\left\{I_{a} \mid a \in A t\right\}\right.$, where $U$ is a finite nonempty set of objects called universe, $A t$ is a finite nonempty set of attributes, $V_{a}$ is a nonempty set of values for an attribute $a \in A t$, and $I_{a}: U \rightarrow V$ is an information function, such that for an object $x \in U$, and a value $v \in V_{a}, I_{a}(x)=v$ means that the object $x$ has the value $v$ on the attribute $a$.

$U=X_{1}, X_{2}, \ldots, X_{n}$ represents the research sample, and $X_{1}, X_{2}, \ldots, X_{n}$ represent the listed companies selected; $F=F_{1}, F_{2}, \ldots, F_{m}$ represents the financial index system, and $F_{1}, F_{2}, \ldots, F_{m}$ represent the financial indexes of listed companies; $D$ is the diction attribute and $d_{i}$ represents the judgment of financial risk, namely, if the financial crisis comes out, $d_{i}=0$, otherwise $d_{i}=1$.

- Step 2: Rough set theory can only address discrete data, however the financial indexes in this paper are continuous data which can not be addressed with knowledge reduction theory. Subsequently, we should discrete the financial data of the registred companies. Through this work, we distinct the financial data with competitive QNN model.

- Step 3: Ownership reduction is to remove independent or trivial possessions under the situation that the classifying capacity of the repository retains the same.

This work adopts discernibility matrix to carry out ownership reduction. We assume that $D S=U, A t, V_{a}, I_{a}$ is the knowledge expressive system, then the discernibility matrix of $S$ is an $n \times n$ matrix.

Given a subset of attributes $A \in A t$, two binary relations between objects on $U$ can be differentiated in an information table and are defined by:

$$
\begin{aligned}
& \operatorname{DIS}(A)=\left\{(x, y) \in U \times U \mid \forall a \in A, I_{a}(x) \neq I_{a}(y)\right\} \\
& I N D(A)=\left\{(x, y) \in U \times U \mid \forall a \in A, I_{a}(x)=I_{a}(y)\right\}
\end{aligned}
$$

A strong discernibility relation $D I S(A)$, states that two objects are strongly discernible with respect to $A$ if they have different values on all attributes in $A$. $A$ strong discernibility relation is irreflexive and symmetric, but not transitive. Besides, a strong indiscernibility relation with respect to $A$ is denoted as $\operatorname{IND}(A)$. Two objects in $U$ satisfy $\operatorname{IND}(A)$ if and only if they have the same values on all attributes in $A$. It can be verified that an indiscernibility relation is reflexive, symmetric and transitive, namely, it is an equivalence relation. It partitions the universe $U$ into a family of disjoint subsets, denoted as $U / I N D(A)=U=\operatorname{IND}(A)=$ 
$\left\{[x]_{I N D(A)} \mid x \in \mathrm{U}\right\}$, where $[x]_{I N D(A)}$ is the A-definable equivalence class containing $x$, i.e.,

$$
[x]_{I N D(A)}=\{y \in \mathrm{U} \mid(x, y) \in I N D(A)
$$

For a subset of attributes, the discernibility and indiscernibility relations can be conveniently expressed in terms of the corresponding relations defined by singleton attribute subsets:

$$
\begin{aligned}
& \operatorname{DIS}(A)=\underset{a \in A}{\cap} \operatorname{DIS}(\{a\}) \\
& \operatorname{IND}(A)=\underset{a \in A}{\bigcap} \operatorname{IND}(\{a\})
\end{aligned}
$$

The discernibility function has the property that all conjunctive normal forms in the infinitesimal disjunctive normal form of function is the reduction of property aggregation $A$.

According to property reduction of decision table of discernibility matrix, it can be divided into five steps to reduce:

Compute discernibility matrix of decision system (table) and establish corresponding extract logic expressions for every list:

\subsection{Structure of QNN model}

The rough set-QNN model the article constructed is a multiple input multiple output model. It has three layers:

The first layer is input layer: The input layer of QNN is the financial indexes from which ineffective financial indexes are eliminate by the algorithm of knowledge reduction.

The second layer is concealed player: Momentum accession and parameter self adaptive algorithm is adopted to solve the problem of slow network convergence and avoid the problem of part least dot under BP QNN algorithm.

If directions of grads remain the same after two times of iteration, it indicates that learning rate descends too slow and that step size should be doubled. But if the directions of grads go opposite, it indicates that learning rate descends excessively and that step size should be halved. By continually adjusting the learning rate, the network convergence speeds up.

The third layer is output layer which is to distinguish companies of financial risk. The output layer of single node can not only simplify the structure of network, but also effectively identify companies of financial risk and avoid making mistakes. The design of single node is applied to the output layer which divide companies into two kinds, namely, financial risk occurring and financial risk not occurring.

\section{Conclusion}

This article has presented a QNN model of and has applied it to the research on the financial risk forecasting of Moroccan companies. We have showed that attribute reduction on financial index of the input of QNN with knowledge reduction of rough set solves the complexity of network structure of standard QNN financial prediction model and lacking in theoretic evidence to ensure the input nods and concealed layers of network, and improves prediction efficiency of the model. Furthermore, we have enhanced the quantum neural network algorithm which reaches well approximation effect.

Subsequently, QNN financial risk model reduces computing time and boost prediction efficiency but not decrease prediction precision. In addition, this article still exist some shortage, such as the sample chosen is relatively less in capacity, which may affect the result of the model in a certain extent, and the choosing of index had limitation too, a large number of nonfinancial factors were not taken into account.

\section{References}

[1] Ezhov, Alexandr., Ventura, Dan. (2000). Quantum neural networks. In: Ed. N. Kasabov, editor, Future Directions for Intelligent

\begin{tabular}{lllll}
\hline 102 & Journal of Information Security Research Volume 10 Number 3 September 2019 \\
\hline
\end{tabular}


Systems and Information Science. Physica-Verlang.

[2] Narayanan, Ajit., Menneer, Tammy. (2000). Quantum artificial neural network architectures and components. In: Information Sciences, $124(1-4), 231-255$.

[3] Altaisky, M. V. (2001). Quantum neural network. Technical report, 2001. http://xxx.lanl.gov/quantph/ 0107012.

[4] Behrman, E. C., Niemel, J., Steck, J. E., Skinner, S. R. (1996). A quantum dot neural network. In: Proceedings of the 4th Workshop on Physics of Computation, 22-24. Boston.

[5] Shafee, Fariel. (2002). Neural networks with c-not gated nodes. Technical report, 2002. http://xxx.lanl.gov/quant-ph/0202016.

[6] Fujita, Yukari., Matsui, Tetsuo. (2002). Quantum gauged neural network: U(1) gauge theory. Technical report, 2002. http:// xxx.lanl.gov/condmat/0207023.

[7] Gupta, S., Zia, R. K. P. (2001). Quantum neural networks. In: Journal of Computer and System Sciences, 63(3) 355-383.

[8] Behrman, E. C., Chandrasheka, V., Wank, Z., Belur, C. K., Steck, J. E., Skinner, S. R. (2002). A quantum neural network computes entanglement. Technical report, 2002. http://xxx.lanl.gov/quantph/ 0202131.

[9] Michael, A., Nielsen, Isaac, L. (2000). Chuang. Quantum computation and quantum information. Cambridge University Press, 2000.

[10] Vedral, V., Plenio, M. B., Rippin, M. A., Knight, P. L. (1997). Quantifying entanglement. In: Physical Review Letters, 78(12) 2275-2279.

[11] Jozsa, R. (1998). Entanglement and quantum computation. In S. Hugget, L. Mason, K. P. Tod, T. Tsou, and N. M. J. Woodhouse, editors, The Geometric Universe, 369-379. Oxford University Press, 1998.

[12] Lov, K., Grover. (1996). A fast quantum mechanical algorithm for database search. In: Proceedings of the 28th ACM STOC, 212-219.

[13] Lov, K. Grover. (1997). Quantum mechanics helps in searching for a needle in a haystack. In: Physical Review Letters, 78, 325-328.

[14] Shor, Peter. (1997). Polynomial-time algorithms for prime factorization and discrete logarithms on a quantum computer. In: SIAM Journal of Computing, 26(5) 1484-1509.

[15] Vedral, Vlatko., Barenco, Adriano., Ekert, Artur. (1996). Quantum networks for elementary arithmetic operations. In: Physical Review A, 54(1) 147-153.

[16] Ventura, Dan., Martinez, Tony. (2000). Quantum associative memory. In: Information Sciences, 124 (1-4) 273-296.

[17] Ezhov, Alexandr. A., Nifanova, Ventura, Dan. (2000). Distributed queries for quantum associative memory. In: Information Sciences, 128 (3-4) 271-293.

[18] Boyer, Michel., Brassard, Gilles., Høyer, Peter., Tapp, Alain. (1996). Tight bounds on quantum searching. In: Proceedings of the Fourth Workshop on Physics and Computation, 36-43.

[19] Blake, C. L., Merz, C. J. (1998). UCI repository of machine learning databases. http://www.ics.uci.edu/mlearn/MLRepository.html. [20] Zarndt, Frederick. (1995). A comprehensive case study: An examination of machine learning and connectionist algorithms. Master's thesis, Brigham Young University.

[21] Edward, Altman, I., Robert, Haldeman, G., Narayanan, P. (1977). ZETA analysis: A new model to identify bankruptcy risk of corporations, Journal of Banking and Finance, North-Holland Publishing Company, 29- 54.

[22] Tsumoto, Shusaku., Tanaka, Hiroshi. (1996). Automated discovery of medical expert system rules from clinical databases based on rough sets, In: Proceeding of Second International Conf. on Knowledge Discovery and Data Mining, USA, 63-69.

[23] Pawlak, Z. (1991). Rough Sets: Theoretical Aspects of Reasoning about Data, Kluwer Academic Publishers, Norwell.

[24] Duntsch, Ivo., Gediga, G. (2000). Rough set data analysis, Encyclopedia of Computer Science and Technology, 281-301

[25] Luba, T., Rybnik, J. (1993). Algorithmic approach to discernibility function with respect to attributes and object reduction, Foundations of Computing and Decision Sciences, 241-258.

[26] Miao, Duoqian., Hou, Lishan. (2004). A comparison of rough set methods and representative inductive learning algorithms, 
Fundamenta Informaticae, IOS Press, 203-219.

[27] Zhang, Hualun. (2006). Establishment of rough fuzzy-ANN model for forecasting enterprise financial distress and its application, Operations Research and Management Science, China, 2006, 103-107.

[28] Dubois, D., Prade, H. (1990). Rough fuzzy sets and fuzzy rough sets, International Journal of General Systems, 1990, 191 209.

[29] Pomykala, J., Pomykala, J. A. (1988). The Stone algebra of rough sets, Bulletin of the Polish Academy of Sciences: Mathematics, Warsaw, 495-508.

[30] Liang, J. Y., Li, D. Y. (2000). Information measures of roughness of knowledge and rough sets in incomplete information systems, Proc. of the Third World Congress on Intelligent Control and Automation, Press of University of Science and Technology of China, Hefei, 2526-2529.

[31] Hu, Xiaohua. (1996). Mining knowledge rules from databases-a rough set approach, In: Proc. of IEEE International Conference on Data Engineering, Los Alamitos, 96-105. 